\title{
Tumor Board Conferences for Multidisciplinary Skin Cancer Management: A Survey of US Cancer Centers
}

\author{
Shoko Mori, BS ${ }^{\mathrm{a}}$; Cristian Navarrete-Dechent, MDª, Tatyana A. Petukhova, MDª Erica H. Lee, MD; \\ Anthony M. Rossi, MD; ; Michael A. Postow, MDc; Lara A. Dunn, MD'; Benjamin R. Roman, MD; \\ Vivian T. Yin, MD; Daniel G. Coit, MD; ; Travis J. Hollmann, MD, PhD ; Klaus J. Busam, $\mathrm{MD}^{\text {h }}$; \\ Kishwer S. Nehal, MDa; and Christopher A. Barker, MD
}

\begin{abstract}
Background: Tumor board conferences (TBCs) are used by oncologic specialists to review patient cases, exchange knowledge, and discuss options for cancer management. These multidisciplinary meetings are often a cornerstone of treatment at leading cancer centers and are required for accreditation by certain groups, such as the American College of Surgeons' Commission on Cancer. Little is known regarding skin cancer TBCs. The objective of this study was to characterize the structure, function, and impact of existing skin cancer TBCs in the United States. Methods: A cross-sectional online survey was administered to physician leaders of skin cancer TBCs at NCl-designated Comprehensive and Clinical Cancer Centers. Results: Of the 59 centers successfully contacted, 14 (24\%) reported not having a conference where skin cancer cases were discussed, and $45(76 \%)$ identified 53 physician leaders. A total of 38 physicians (72\%) completed the survey. Half of the meeting leaders were medical and/or surgical oncologists, and dermatologists led one-third of meetings. TBCs had a moderate to significant impact on patient care according to $97 \%$ of respondents. All respondents indicated that the meetings enhanced communication among physicians and provided an opportunity for involved specialists and professionals to discuss cases. The most frequently cited barrier to organizing TBCs was determining a common available date and time for attendees (62\%). The most common suggestion for improvement was to increase attendance, specialists, and/or motivation. Conclusions: Results showed overall consistency in meeting structure but variability in function, which may be a reflection of institutional resources and investment in the conference. Future directions include defining metrics to evaluate changes in diagnosis or management plan after tumor board discussion, attendance, clinical trial enrollment, and cost analysis. Results of this survey may aid other institutions striving to develop and refine skin cancer TBCs.
\end{abstract}

Skin cancer is the most common form of cancer in the United States. The incidence of nonmelanoma skin cancer (NMSC), such as basal cell and squamous cell carcinomas, has increased from an estimated 3.5 mil-

\footnotetext{
aDermatology Service, Department of Medicine, Memorial Sloan Kettering Cancer Center, New York, New York; 'bepartment of Dermatology, Facultad de Medicina, Pontificia Universidad Católica de Chile, Santiago, Chile; and 'Melanoma and Immunotherapeutics Oncology Service, Department of Medicine, ${ }^{\mathrm{d}} \mathrm{Head}$ and Neck Oncology Service, Department of Medicine eHead and Neck Service, Department of Surgery, ${ }^{\dagger}$ Ophthalmic Oncology Service, Department of Surgery, ${ }^{9}$ Gastric and Mixed Tumor Service, Department of Surgery, hDepartment of Pathology, and 'Department of Radiation Oncology, Memorial Sloan Kettering Cancer Center, New York, New York. Submitted February 8, 2018; accepted for publication May 7, 2018. Dr. Postow has disclosed that he is on the scientific advisory board for NewLink Genetics, Bristol-Myers Squibb, Novartis, Merck, Array BioPharma, and Incyte, and that he receives honoraria from Bristol-Myers Squibb and Merck. Dr. Yin has disclosed that she is a consultant for Merz Pharmaceuticals and Genentech. Dr. Barker has disclosed that he is on the scientific
}

lion cases in 2006 to 5.4 million cases in 2012.1,2 Additionally, the incidence of melanoma and Merkel cell carcinoma is also on the rise. ${ }^{3,4}$ Most skin cancers are detected early and are easily cured with a single

advisory board for Pfizer and has received grant/research support from Merck, Bristol-Myers Squibb, Elekta, and Amgen. The remaining authors have disclosed that they have no financial interests, arrangements, affiliations, or commercial interests with the manufacturers of any products discussed in this article or their competitors.

This research is funded in part by a grant from the $\mathrm{NCl} / \mathrm{NIH}$ (P30-CA008748) made to Memorial Sloan Kettering Cancer Center.

Author contributions: Data acquisition, analysis, and interpretation: All authors. Manuscript preparation and critical revision: All authors. Final approval: All authors.

Correspondence: Christopher A. Barker, MD, Department of Radiation Oncology, Memorial Sloan Kettering Cancer Center, 1250 First Avenue, New York, NY 10065. Email: barkerc@mskcc.org 
treatment modality. However, a subset of patients present with recurrent and/or advanced skin cancer and require the expertise of several specialists for optimal management. Multidisciplinary team meetings, also known as tumor board conferences (TBCs), provide an opportunity to discuss the full spectrum of diagnostic, therapeutic, and social issues related to individual patients and to develop a coordinated plan for patients with complex skin cancer. ${ }^{5}$

Multidisciplinary management is often a cornerstone of treatment at leading cancer centers. In fact, a multidisciplinary TBC is required for accreditation by the American College of Surgeons' Commission on Cancer. ${ }^{6}$ In other countries, such as the United Kingdom, multidisciplinary team meetings are an essential component of the national standard for cancer care. ${ }^{7}$ Multidisciplinary TBCs have demonstrated value in the management of patients with various cancers, including breast, gynecologic, thoracic, and upper gastrointestinal malignancies. ${ }^{8-11}$ TBCs provide an opportunity to discuss treatment options among different specialists who offer a range of perspectives and management approaches. A study on the impact of a breast cancer TBC found that management plans are commonly modified to reflect the most current data and literature. ${ }^{8}$ TBCs also encourage adherence to clinical practice guidelines, improve communication among physicians, and provide a forum for continuing medical education. ${ }^{12}$

Despite the general benefits of TBCs, there is a paucity of published information regarding the structure and function of these multidisciplinary meetings among skin cancer specialists. The current study aimed to characterize existing skin cancer TBCs at NCI-designated Cancer Centers across the United States, with the goal of better understanding institutional practices across the country.

\section{Methods}

In a cross-sectional study design, an online survey was created through SurveyMonkey. The survey consisted of 26 questions that were formulated after a comprehensive literature review and discussion among the authors (supplemental eAppendix 1 , available with this article at JNCCN.org). Questions were grouped into 3 categories: (1) structure, (2) function, and (3) impact. Structure included information regarding the attendees and format of the
TBC. Function was defined as conference activities and actions. Questions regarding impact addressed the value of the conference, as well as barriers. The survey was vetted internally by a multidisciplinary group of physicians involved in skin cancer management at Memorial Sloan Kettering Cancer Center (MSKCC). The study was determined to be exempt from review by the Institutional Review Board of MSKCC.

In 2017, the top 4\% of cancer research institutions $(n=69)$ were recognized as NCI-designated Cancer Centers. A subset of these institutions $(n=49)$ also demonstrate expertise in transdisciplinary research, earning the designation of a Comprehensive Cancer Center. ${ }^{13} \mathrm{NCCN}$ is another alliance of 27 leading US cancer centers that have "pioneered the concept of the multidisciplinary team approach to patient care."14 Of the 69 NCI-designated Cancer Centers, 7 are Basic Laboratory Cancer Centers that do not provide patient treatment, and therefore were not included in the study. The remaining 62 centers (including all 27 NCCN Member Institutions) were contacted initially by telephone to determine whether they had an existing skin cancer TBC. If an institution had $\geq 1$ skin cancer TBC, the primary physician leader of the tumor board was administered the survey by email. The survey was emailed up to 3 times to maximize response rate. If there was no response after the third attempt, the center was excluded.

\section{Results}

\section{Target Study Population}

Of the 62 NCI-designated Comprehensive and Clinical Cancer Centers, including our own institution, we were unable to contact 3 centers $(5 \%)$ despite multiple attempts. Of the 59 centers successfully contacted, 14 (24\%) reported not having a conference where skin cancer cases were discussed. Three centers $(5 \%)$ did not have skin cancer-specific TBCs, but did discuss skin cancer cases in a conference along with other cancers (eg, sarcoma, head and neck cancer) and therefore were included in the study; 7 cancer centers (12\%) had $>1$ skin cancer TBC (eg, a melanoma and an NMSC TBC).

A total of 45 institutions identified 53 physician leaders for skin cancer conferences. To be included in the study analysis, at least $80 \%$ of the survey questions had to be completed. Of the 53 physician lead- 
ers, 38 (72\%) completed the survey and were included in the final analysis.

\section{Conference Structure}

Table 1 summarizes survey responses regarding conference structure. The geographic distribution, according to the census regions identified by the US Census Bureau, was relatively equal among the survey respondents. Most respondents (87\%) indicated that the meeting had a designated leader. Half of the respondents $(52 \%)$ indicated that specialists from multiple clinical disciplines served as group

\begin{tabular}{|c|c|}
\hline \multicolumn{2}{|l|}{ Geographic location } \\
\hline Northeast & $9(24 \%)$ \\
\hline South & $10(26 \%)$ \\
\hline Midwest & $8(21 \%)$ \\
\hline West & $11(29 \%)$ \\
\hline \multicolumn{2}{|l|}{ Designated meeting leader } \\
\hline Yes & $33(87 \%)$ \\
\hline No & $5(13 \%)$ \\
\hline \multicolumn{2}{|c|}{ Designated meeting secretary or coordinator } \\
\hline Yes & $32(84 \%)$ \\
\hline No & $6(16 \%)$ \\
\hline \multicolumn{2}{|l|}{ Number of attendees } \\
\hline $1-5$ & $7(18 \%)$ \\
\hline $6-10$ & $18(47 \%)$ \\
\hline $11-15$ & $13(34 \%)$ \\
\hline $16-20$ & $0(0 \%)$ \\
\hline $21-30$ & $0(0 \%)$ \\
\hline$>30$ & $0(0 \%)$ \\
\hline \multicolumn{2}{|c|}{ Duration of establishment, $y$} \\
\hline$<1$ & $0(0 \%)$ \\
\hline $1-3$ & $5(13 \%)$ \\
\hline $4-6$ & $7(18 \%)$ \\
\hline $7-10$ & $6(16 \%)$ \\
\hline $11-15$ & $7(18 \%)$ \\
\hline$>15$ & $13(34 \%)$ \\
\hline \multicolumn{2}{|l|}{ Frequency of meeting } \\
\hline Weekly & $17(45 \%)$ \\
\hline Twice per month & $14(37 \%)$ \\
\hline Monthly & $6(16 \%)$ \\
\hline As needed & $1(3 \%)$ \\
\hline \multicolumn{2}{|l|}{ Duration of meeting, $\mathrm{min}$} \\
\hline 30 & $2(5 \%)$ \\
\hline 60 & $28(74 \%)$ \\
\hline 90 & $7(18 \%)$ \\
\hline 120 & $1(3 \%)$ \\
\hline \multicolumn{2}{|c|}{ Number of cases discussed per meeting } \\
\hline $1-5$ & $13(34 \%)$ \\
\hline $6-10$ & $11(29 \%)$ \\
\hline $11-15$ & $10(26 \%)$ \\
\hline $16-20$ & $1(3 \%)$ \\
\hline$>20$ & $3(8 \%)$ \\
\hline \multicolumn{2}{|l|}{ Videoconferencing used } \\
\hline Yes & $22(58 \%)$ \\
\hline No & $16(42 \%)$ \\
\hline \multicolumn{2}{|c|}{ Financial support of meeting } \\
\hline Institution & $24(63 \%)$ \\
\hline Individual departments & $20(53 \%)$ \\
\hline Philanthropic source & $9(24 \%)$ \\
\hline
\end{tabular}

coleaders, whereas $48 \%$ indicated the leaders were from a single specialty. The distribution of the clinical specialties for group leaders is shown in Figure 1. Medical and surgical oncologists together constituted half of the meeting leaders. Dermatologists, including dermatologic surgeons and dermatopathologists, comprised roughly one-third of the leaders. Most respondents (84\%) also indicated that a meeting secretary or coordinator was involved in the meeting. The distribution of attendees (and clinical specialists) attending the meeting is shown in Figure $2 ;>90 \%$ of meetings were attended by medical and/ or surgical oncologists. TBCs most often occurred weekly (45\%) and lasted for 1 hour (74\%). Videoconferencing was used by $58 \%$ of respondents. Most meetings were financially supported by the institution or individual departments, and 24\% reported funding from a philanthropic source.

\section{Conference Function}

A summary of the survey results regarding meeting function is shown in Table 2. Melanoma and Merkel cell carcinoma (92\% for both) were the skin cancers that most groups discussed, followed by cutaneous squamous cell carcinoma (84\%). All meetings involved presentation of clinical history, and most also presented radio-

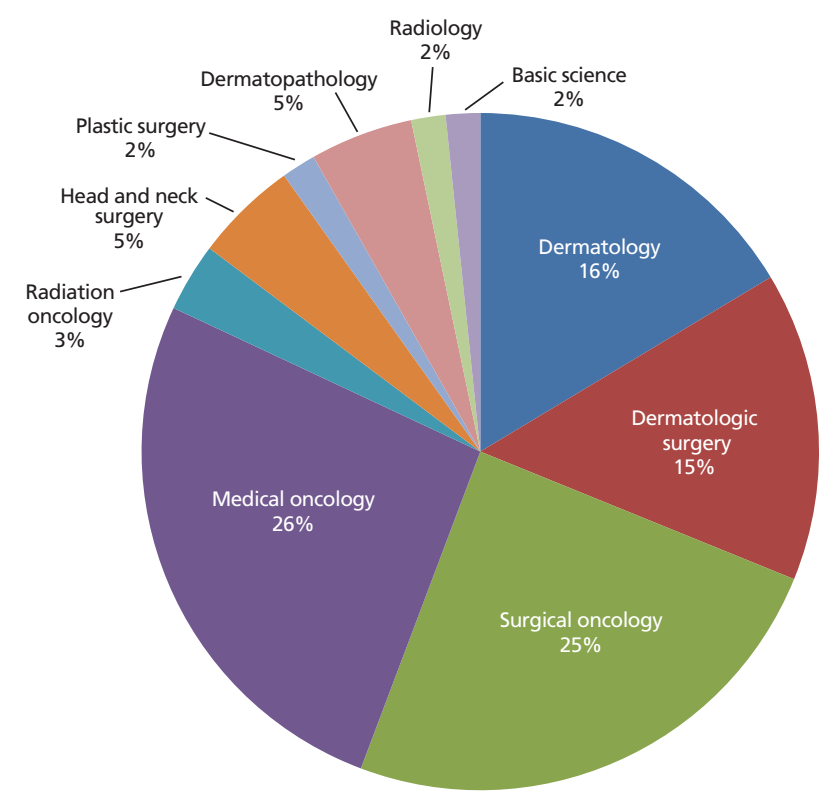

Figure 1. Clinical specialties of skin cancer tumor board conference leaders $\left(\mathrm{N}=61^{\mathrm{a}}\right)$.

a Of the $33 / 38(87 \%)$ respondents who indicated a designated meeting leader, 16 indicated a single specialty and 17 indicated multiple specialties as leaders, resulting in 61 leaders among 10 specialties. 
A

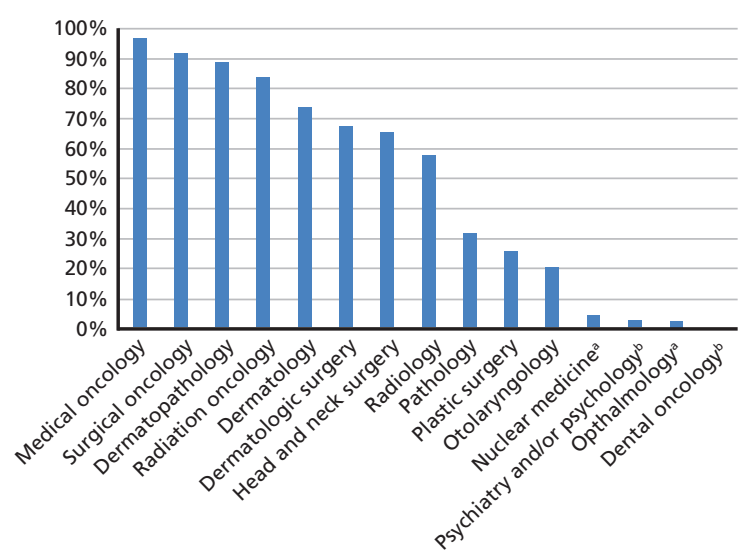

B

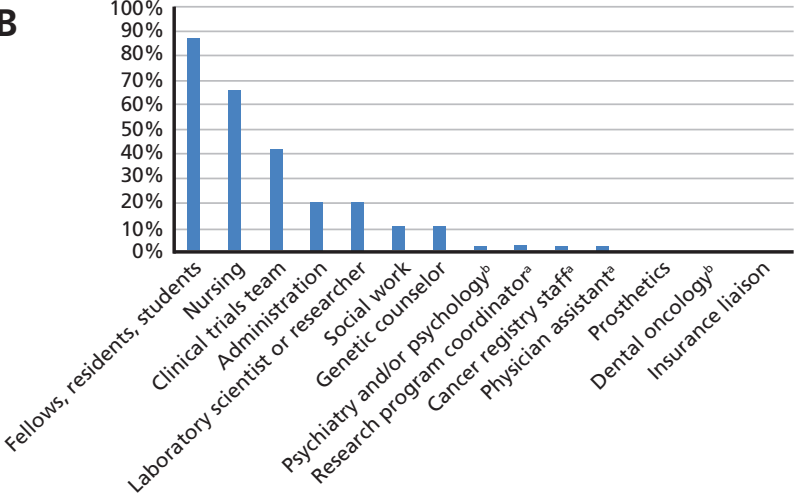

Figure 2. Distribution of (A) physician and (B) nonphysician attendees of skin cancer tumor boards. Percentage on the vertical axis represents the survey respondents indicating the specified group as attending their meeting.

answer was written in by $\geq 1$ respondents under the "other" option, and was not listed as one of the multiple choice options.

${ }^{b}$ Specialties that may include both physician and nonphysician individuals.

graphic images (95\%), histopathology images (92\%), and clinical photos (87\%). Molecular pathology and genetic test results were presented less frequently (74\% and $61 \%$, respectively). Half of the respondents indicated that radiographic images were at least sometimes reviewed by a radiologist. Research protocols were discussed in $61 \%$ of meetings, and research presentations or guest speakers were featured in 39\% of meetings. Physicians followed up with patients after the meeting according to $76 \%$ of respondents. The TBC led to further discussion among physicians according to $71 \%$ of respondents. Notes were recorded in the patient's medical record by $58 \%$ of respondents, whereas $24 \%$ of meetings had minutes recorded. A database of patients with skin cancer was maintained by $68 \%$ of the groups.

\section{Conference Impact}

Results regarding the impact of TBCs are shown in Table 3. A total of $97 \%$ of respondents indicated

\begin{tabular}{|c|c|}
\hline \multicolumn{2}{|l|}{ Diagnoses reviewed } \\
\hline Melanoma & $35(92 \%)$ \\
\hline Cutaneous squamous cell carcinoma & $32(84 \%)$ \\
\hline Basal cell carcinoma & $31(82 \%)$ \\
\hline Merkel cell carcinoma & $35(92 \%)$ \\
\hline Cutaneous adnexal neoplasm & $31(82 \%)$ \\
\hline Dermatofibrosarcoma protuberans & $27(71 \%)$ \\
\hline Cutaneous lymphoma & $8(21 \%)$ \\
\hline \multicolumn{2}{|l|}{ Type of cases } \\
\hline Not yet diagnosed & $22(58 \%)$ \\
\hline Presurgical & $34(89 \%)$ \\
\hline Postsurgical & $37(97 \%)$ \\
\hline Challenging cases & $38(100 \%)$ \\
\hline Recurrent disease & $36(95 \%)$ \\
\hline Follow-up & $23(61 \%)$ \\
\hline Interest & $31(82 \%)$ \\
\hline Potential clinical trial enrollment ${ }^{a}$ & $2(5 \%)$ \\
\hline Monitor response to treatment ${ }^{\mathrm{a}}$ & $1(3 \%)$ \\
\hline \multicolumn{2}{|l|}{ Type of information presented } \\
\hline Clinical history & $38(100 \%)$ \\
\hline Clinical photos & $33(87 \%)$ \\
\hline Radiographic imaging & $36(95 \%)$ \\
\hline Histopathology slides & $35(92 \%)$ \\
\hline Molecular pathology & $28(74 \%)$ \\
\hline Genetic testing & $23(61 \%)$ \\
\hline Available clinical trials ${ }^{a}$ & $1(3 \%)$ \\
\hline Confocal imaging ${ }^{a}$ & $1(3 \%)$ \\
\hline Literature reviewa $^{a}$ & $1(3 \%)$ \\
\hline \multicolumn{2}{|l|}{ Radiographic images reviewed by a radiologist } \\
\hline Yes & $13(34 \%)$ \\
\hline No & $19(50 \%)$ \\
\hline Sometimes & $6(16 \%)$ \\
\hline \multicolumn{2}{|c|}{ Histopathology slides reviewed by a dermatopathologist } \\
\hline Yes & $31(82 \%)$ \\
\hline No & $2(5 \%)$ \\
\hline Sometimes & $5(13 \%)$ \\
\hline \multicolumn{2}{|l|}{ Discussion of research protocols } \\
\hline Yes & $23(61 \%)$ \\
\hline No & $15(39 \%)$ \\
\hline \multicolumn{2}{|l|}{ Research talks/guest speakers } \\
\hline Yes & $15(39 \%)$ \\
\hline No & $23(61 \%)$ \\
\hline \multicolumn{2}{|l|}{ Follow-up action after meeting } \\
\hline Minutes recorded & $9(24 \%)$ \\
\hline Notes recorded in patient's chart & $22(58 \%)$ \\
\hline Physician contact with patient & $29(76 \%)$ \\
\hline Nursing/PA/ancillary staff contact with patient & $21(55 \%)$ \\
\hline Further discussion among physicians & $27(71 \%)$ \\
\hline Cases entered in a database & $9(24 \%)$ \\
\hline No further contact & $5(13 \%)$ \\
\hline \multicolumn{2}{|l|}{ Does your program manage a skin cancer database? } \\
\hline Yes & $26(68 \%)$ \\
\hline No & $12(32 \%)$ \\
\hline
\end{tabular}

Abbreviation: PA, physician assistant.

"Answer was written in by $\geq 1$ respondents under the "other" option, and was not listed as one of the multiple choice options.

that their TBCs had a moderate to significant impact on patient care. All respondents indicated that the meetings enhanced communication among physicians, and was an opportunity for physicians, nurses, and allied health professionals to discuss cases together. Treatment options that were not previously 
Tumor Board Conferences for Skin Cancer

\begin{tabular}{|c|c|}
\hline \multicolumn{2}{|l|}{ Impact of meeting on patient care } \\
\hline None & $0(0 \%)$ \\
\hline Minimal & $1(3 \%)$ \\
\hline Moderate & $12(32 \%)$ \\
\hline Significant & $24(65 \%)$ \\
\hline \multicolumn{2}{|l|}{ Value of meeting } \\
\hline Enhanced communication among physicians & $37(100 \%)$ \\
\hline Continuing medication education for physicians & $27(73 \%)$ \\
\hline Encouraged use of most recent guidelines for management & $29(78 \%)$ \\
\hline More treatment options for patients & $34(92 \%)$ \\
\hline Reduced number of visits for patients & $15(41 \%)$ \\
\hline $\begin{array}{l}\text { Opportunity for physicians, nurses, allied health } \\
\text { professionals to discuss cases together }\end{array}$ & $37(100 \%)$ \\
\hline Drives development of new research protocols & $24(65 \%)$ \\
\hline Increased enrollment of patients in clinical trials & $25(68 \%)$ \\
\hline Improved standardization of care ${ }^{a}$ & $2(5 \%)$ \\
\hline \multicolumn{2}{|l|}{ Barriers to setting up meeting } \\
\hline Finding a common date and time & $23(62 \%)$ \\
\hline Lack of administrative support & $7(19 \%)$ \\
\hline Lack of specialties & $9(24 \%)$ \\
\hline Finding a physician to take on leadership role & $3(8 \%)$ \\
\hline Lack of time to prepare & $9(24 \%)$ \\
\hline High caseload & $8(22 \%)$ \\
\hline Lack of financial support & $13(35 \%)$ \\
\hline None of the above & $6(16 \%)$ \\
\hline Lack of technological support ${ }^{\mathrm{a}}$ & $1(3 \%)$ \\
\hline Time constraints of meeting ${ }^{a}$ & $1(3 \%)$ \\
\hline Competing tumor boards ${ }^{a}$ & $1(3 \%)$ \\
\hline
\end{tabular}

aAnswer was written in by $\geq 1$ respondents under the "other" option, and was not listed as one of the multiple choice options.

considered were discussed in $92 \%$ of meetings, and $78 \%$ indicated that the meeting encouraged use of the most recent guidelines for management.

The most frequently cited barriers to organizing TBCs was determining a common available date and time for attendees $(62 \%)$ and lack of financial support $(35 \%)$. The most common suggestion for improvement was to increase attendance, specialties, and/or motivation to attend. One respondent specifically indicated that they would like community providers to attend the meeting as well. Five respondents suggested the implementation of a skin cancer database. Other suggestions were the inclusion of radiographic images or attendance by a radiologist, increased financial support, the provision of food at the meeting, and increased administrative support. Improved documentation of the meeting was also suggested by 3 respondents.

\section{Discussion}

In the United States, the NCCN Clinical Practice Guidelines in Oncology (NCCN Guidelines) recommend multidisciplinary tumor board consultation for complicated and high-risk skin cancer cases. ${ }^{15-17}$ However, it is not specifically stated what kind of format this tumor board should take. Furthermore, there is a paucity of information regarding skin cancer TBCs, both within the medical literature and on the websites of individual cancer centers. Our survey findings provide relevant information regarding the structure, function, and impact of existing skin cancer TBCs at cancer centers across the United States. Among existing TBCs, there was relative consistency in the meeting structure. There was heterogeneity in conference function, which may be explained by the institutions' available resources and their investment in the TBC or skin cancer program. Remarkably, we observed that nearly a quarter of NCI-designated Comprehensive and Clinical Cancer Centers did not have a TBC in which skin cancer cases were discussed, either as a primary focus or included among discussions of other cancers. This finding highlights the importance of defining the purpose and structure of skin cancer TBCs for institutions that hope to start their own conference, as well as for patients who would benefit from multidisciplinary discussion and the providers who may refer them.

A model for standardization can be seen in the United Kingdom, where there is a greater availability of published information regarding multidisciplinary skin cancer management. The United Kingdom National Multidisciplinary Guidelines for the management of NMSC recommend high-risk patients to be treated by a multidisciplinary skin cancer team. ${ }^{18}$ More specifically, the National Institute for Health and Care Excellence (NICE), which publishes national health guidance for England, recommends 2 levels of multidisciplinary teams for the management of patients with skin cancer: one for local hospitals and one for specialized cancer centers. These guidelines, which are based on best available evidence and consensus positions, describe the specialties that should be represented, types of cases to be presented, frequency of meetings, and activities that should take place, including the recommended auditing of skin cancer cases on a regular basis. ${ }^{19}$ Adherence to these guidelines, as well as costs associated with their implementation, will require further investigation. However, this model may serve as a starting point for tumor board standardization.

The principal metric of the impact of TBCs is the probability of change in individual patient man- 
Mori et al

agement after formal multidisciplinary discussion. Most survey respondents (97\%) indicated that their conference had a moderate to significant impact on patient care. As reported in the literature, patients discussed at multidisciplinary TBCs may receive a change in diagnosis $(18.4 \%-29 \%)$ or treatment plan $(20 \%-52 \%) .{ }^{8-10,20}$ TBCs can improve coordination of patient care and clinical decision-making, and increase adherence to nationally accepted guidelines. ${ }^{11,21}$ The multidisciplinary setting of these meetings facilitates exchange of perspectives from different specialties and can lead to a more comprehensive discussion of each patient's treatment options. The current survey findings are consistent with these previously described benefits of TBCs. Documentation of tumor board discussion and follow-up of patients showed variability among survey respondents. These are essential areas in which standardization would be particularly beneficial in assessing meeting impact.

Costs were not directly assessed in the current survey; however, $41 \%$ of respondents indicated that tumor board discussions led to reduced clinic visits for patients. A cost analysis study found that patients treated in a multidisciplinary melanoma clinic saved a third-party payer roughly $\$ 1,600$ per patient compared with those treated in the community. ${ }^{22}$ The lower costs were attributed to fewer office visits, blood tests, radiographic imaging, and procedures. It is also important to note the institutional costs required to maintain multidisciplinary meetings. An analysis of multidisciplinary team meetings in the United Kingdom found that the median cost per month of these meetings, based on attendees' salaries and basic overhead costs, was $£ 5,136$, which is roughly USD $\$ 7,277 .{ }^{23}$ Beyond consideration of salary support, most respondents indicated that TBCs were funded by the institution and/or individual departments. Research funds or grants were not included as answer options; however, these could be a potential source of funding for TBCs that use the meeting for research purposes.

These results suggest that institutions should do more to support a skin cancer TBC. Studies have shown that physicians affiliated with accredited cancer centers are more likely to participate in TBCs. ${ }^{24}$ However, participation can be time-consuming, and therefore viewed as unfavorable by prospective attendees. Nearly a quarter of our survey respondents indicated lack of time to prepare for the meeting as a barrier. Other common barriers included determining a common date and time for the meeting (62\%) and lack of financial support (35\%). A systematic review of multidisciplinary cancer teams found that insufficient time for preparation, excessive caseload, low attendance, lack of nursing input, and paucity of leadership affect management decisions. ${ }^{25} \mathrm{~A}$ study from the United Kingdom reported that the most frequently cited organizational improvement to multidisciplinary team working was to have more time dedicated to the meeting. ${ }^{26}$ In a follow-up analysis of these same survey results, the authors found that good relationships between team members, adequate skills in communication and leadership, and organizational support were key factors for effective team working. ${ }^{27}$ Further research into the appropriate amount and type of institutional support for the maintenance of an effective multidisciplinary skin cancer program is clearly needed.

There are several limitations to this study. One is that we may not have captured all TBCs in which skin cancers are discussed, resulting in an underestimation of the true number of existing conferences. Another limitation is the inherent bias in those who responded to the survey. It is possible that those who did not respond to the survey were less satisfied with the structure or function of their TBC. In addition, the survey population consists of a select group of physicians who are part of nationally recognized cancer centers. The findings of this study may not be generalizable to all centers that treat patients with skin cancer. In fact, further investigation into barriers to multidisciplinary care as well as the role of community hospitals and their inclusion in TBCs are critical in developing a greater understanding of this form of care. The survey questions also focused on the clinical aspect of TBCs. Although most respondents indicated that these TBCs resulted in changes in patient management, the magnitude of change and the percentage of patients with change were not captured. Many TBCs function as both a conduit for clinical care in a multidisciplinary setting and an arena to facilitate research. The survey did not comprehensively assess the research component of TBCs. Lastly, some of the survey questions included an "other" option where respondents wrote in an answer. If those answers were included in the multiple-choice options, there may have been a greater number of respondents also choosing these answers. 
Future directions for research include defining metrics for skin cancer TBCs. These metrics may include reasons for presentation, changes in diagnosis or management plan following tumor board discussion, attendance, enrollment in clinical trials, and cost analysis. In addition, the optimal method in which to record the proceedings of the meeting, such as formal minutes, documentation in the patient's medical record, or a tumor board database, remains to be determined. Although assessing the value of TBCs is difficult, standardizing the structure and recording these key metrics may be a step toward further understanding the potential impact of these multidisciplinary meetings.

\section{Conclusions}

Nearly one-quarter of NCI-designated Comprehensive and ClinicalCancerCenters did not report having a TBC where skin cancer cases were discussed. Existing skin cancer TBCs show relative homogeneity in structure, but variability in function. Most respondents indicated moderate to significant impact on patient care, but further research into the objective value of skin cancer multidisciplinary meetings is needed. Existing conferences enhance communication among physicians and provide a valuable opportunity for involved specialties and professions to discuss cases. The results of this survey may aid other institutions striving to develop and refine skin cancer TBCs.

\section{References}

1. Rogers $H W$, Weinstock $M A$, Harris $A R$, et al. Incidence estimate of nonmelanoma skin cancer in the united states, 2006. Arch Dermatol 2010;146:283-287.

2. Rogers HW, Weinstock MA, Feldman SR, Coldiron BM. Incidence estimate of nonmelanoma skin cancer (keratinocyte carcinomas) in the US population, 2012. JAMA Dermatol 2015;151:1081-1086.

3. Fitzgerald TL, Dennis S, Kachare SD, et al. Dramatic increase in the incidence and mortality from Merkel cell carcinoma in the United States. Am Surg 2015;81:802-806.

4. American Cancer Society. Key Statistics for Melanoma Skin Cancer. Available at: https://www.cancer.org/cancer/melanoma-skin-cancer/about/keystatistics.html. Accessed December 28, 2017.

5. National Cancer Institute. NCI Dictionary of Cancer Terms: Tumor Board Review. Available at: https://www.cancer.gov/publications/dictionaries/cancer-terms?cdrid=322893. Accessed August 30, 2017.

6. American College of Surgeons/Commission on Cancer. Cancer Program Standards: Ensuring Patient-Centered Care. 2016 Edition. Available at: https://www.facs.org/quality-programs/cancer/coc/standards. Accessed $\mathrm{Au}$ gust 30, 2017.

7. National Cancer Peer Review-National Cancer Action Team. National Cancer Peer Review Programme. Manual for Cancer Services: Acute Oncology - Including Metastatic Spinal Cord Compression Measures, version 1.0. Available at: https://assets.publishing.service.gov.uk/government/uploads/ system/uploads/attachment_data/file/216121/dh_125889.pdf. Accessed August 30, 2017.

8. Newman EA, Guest AB, Helvie MA, et al. Changes in surgical management resulting from case review at a breast cancer multidisciplinary tumor board. Cancer 2006;107:2346-2351.

9. Greer HO, Frederick PJ, Falls NM, et al. Impact of a weekly multidisciplinary tumor board conference on the management of women with gynecologic malignancies. Int J Gynecol Cancer 2010;20:1321-1325.

10. van Hagen $P$, Spaander MC, van der Gaast A, et al. Impact of a multidisciplinary tumour board meeting for upper-GI malignancies on clinical decision making: a prospective cohort study. Int J Clin Oncol 2013;18:214-219.

11. Freeman RK, Van Woerkom JM, Vyverberg A, Ascioti AJ. The effect of a multidisciplinary thoracic malignancy conference on the treatment of patients with lung cancer. Eur J Cardiothorac Surg 2010;38:1-5.

12. El-Saghir NS, Chahara RN, Kreidieh FY, et al. Global practice and efficiency of multidisciplinary tumor boards: results of an American Society of Clinical Oncology international survey. J Glob Oncol 2015;1:57-64.

13. National Cancer Institute. NCI-Designated Cancer Centers. Available at: https://www.cancer.gov/research/nci-role/cancer-centers. Accessed December $8,2017$.

14. National Comprehensive Cancer Network. About NCCN. Available at: https://www.nccn.org/about/. Accessed December 29, 2017.

15. Bichakjian CK, Aai SZ, Alam M, et al. NCCN Clinical Practice Guidelines in Oncology: Basal Cell Skin Cancer. Version 1.2019. Accessed September 10, 2018. To view the most recent version of these guidelines, visit NCCN.org.

16. Bichakjian CK, Aai SZ, Alam M, et al. NCCN Clinical Practice Guidelines in Oncology: Squamous Cell Skin Cancer. Version 1.2019. Accessed September 10, 2018. To view the most recent version of these guidelines, visit NCCN.org.

17. Coit DG, Thompson JA, Albertini MR, et al. NCCN Clinical Practice Guidelines in Oncology: Melanoma. Version 3.2018. Accessed September 10,2018 . To view the most recent version of these guidelines, visit NCCN. org

18. Newlands C, Currie R, Memon A, et al. Non-melanoma skin cancer: United Kingdom National Multidisciplinary Guidelines. J Laryngol Otol 2016;130(Suppl 2):S125-132.

19. National Institute for Health and Care Excellence. Improving Outcomes for People With Skin Tumours Including Melanoma. Available at: https://www. nice.org.uk/guidance/csg8. Accessed August 30, 2017.

20. Basta YL, Bolle S, Fockens P, Tytgat KM. The value of multidisciplinary team meetings for patients with gastrointestinal malignancies: a systematic review. Ann Surg Oncol 2017;24:2669-2678.

21. Taylor C, Ramirez AJ. Multidisciplinary team members' views about MDT working: results from a survey commissioned by the National Cancer Action Team. National Cancer Action Team, 2009. Available at: www.ncin.org.uk/ mdt. Accessed August 30, 2017.

22. Fader DJ, Wise CG, Normolle DP, Johnson TM. The multidisciplinary melanoma clinic: a cost outcomes analysis of specialty care. J Am Acad Dermatol 1998;38:742-751.

23. Deleso PB, Coward JI, Letsa I, et al. A study of the decision outcomes and financial costs of multidisciplinary team meetings (MDMs) in oncology. $\mathrm{Br} \mathrm{J}$ Cancer 2013;109:2295-2300.

24. Scher KS, Tisnado DM, Rose DE, et al. Physician and practice characteristics influencing tumor board attendance: results from the provider survey of the Los Angeles women's health study. J Oncol Pract 2011;7:103-110.

25. Lamb BW, Brown KF, Nagpal K, et al. Quality of care management decisions by multidisciplinary cancer teams: a systematic review. Ann Surg Oncol 2011;18:2116-2125.

26. Lamb BW, Sevdalis N, Arora S, et al. Teamwork and team decision-making in multidisciplinary cancer conferences: barriers, facilitators, and opportunities for improvement. World J Surg 2011;35:1970-1976.

27. Lamb BW, Taylor C, Lamb JN, et al. Facilitators and barriers to teamworking and patient centeredness in multidisciplinary cancer teams: findings of a national study. Ann Surg Oncol 2013;20:1408-1416. 